\title{
Diplopia as the First Sign of Gastric Carcinoma
}

\author{
Suzanna L. Roohéa ${ }^{\text {Ivan M. Gan }}{ }^{b} \quad$ Kim van der Weerd ${ }^{c} \quad$ Boaz Lopuhaäd \\ Robert M. Verdijk ${ }^{d}$ Dion Paridaens ${ }^{a, e}$ \\ aDepartment of Oculoplastic and Orbital Surgery, The Rotterdam Eye Hospital, Rotterdam, \\ The Netherlands; ${ }^{b}$ Department of Ophthalmology, Franciscus Gasthuis \& Vlietland, \\ Rotterdam, The Netherlands; 'Department of Internal Medicine, Franciscus Gasthuis \& \\ Vlietland, Rotterdam, The Netherlands; ${ }^{d}$ Department of Pathology, Erasmus University

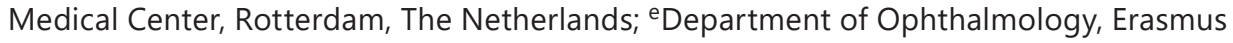 \\ University Medical Center, Rotterdam, The Netherlands
}

\section{Keywords}

Diplopia · Gastric carcinoma · Orbital metastases - Extraocular muscle

\begin{abstract}
Orbital metastasis may be the initial manifestation of a malignancy of unknown origin. The primary locations of orbital metastasis are usually the lung, prostate, gastrointestinal tract, skin, kidney, eye, or thyroid gland. Metastasis of gastric carcinoma to an extraocular eye muscle is extremely rare. A solitary thickening in an extraocular eye muscle with no inflammatory features is suspect for a tumor. Symptoms such as diplopia, proptosis, ptosis, vision loss, or pain may be associated with an orbital malignancy. Our patient, a 67-year-old man known with radically resected prostate cancer, presented with complaints of vertigo with a tendency to fall, headache, and diplopia when looking to the right. As a coincidental finding, swelling of the rectus lateralis muscle of the left eye was observed on imaging. Extensive additional investigations showed that a gastric carcinoma with intraorbital and leptomeningeal metastasis was the cause. In conclusion, a solitary thickened extraocular eye muscle should be recognized in time and examined further.
\end{abstract}

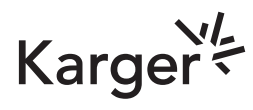




\section{Introduction}

This case report demonstrates that adult-onset diplopia may be caused by metastasis to the extraocular eye muscles. The orbital metastasis turned out to be a rare initial manifestation of a malignancy of a yet unknown origin.

Our patient presented with vertigo, headache, and diplopia accompanied by complains of loss of appetite and aversion to meat. As a coincidental finding, imaging of the eye showed noninflammatory swelling of the rectus lateralis muscle of the left eye, suspicious for a malignant lesion. Further investigations demonstrated that the swelling of the rectus lateralis muscle was caused by a metastasis from gastric cancer.

\section{Case Presentation}

The patient, a 67-year-old man known with radically removed prostate cancer, presented with complaints of vertigo with a tendency to fall, headache, and diplopia when looking to the right. Initially, a cerebrovascular incident was suspected for which the patient was sent to the neurologist. However, the department of Neurology did not find a neurological explanation for the complaints. Cerebral ischemia and bleeding were excluded, but a swelling of the rectus lateralis muscle of the left eye was observed on the brain MRI (shown in Fig. 1). For this, the local ophthalmologist referred the patient to the department of Oculoplastic and Orbital Surgery at The Rotterdam Eye Hospital.

Ophthalmological examination at our clinic revealed a slight right torticollis in combination with an adduction limitation of the left eye (shown in Fig. 2a-c). No significant proptosis of the left eye was measured. No abnormalities were found during the slit-lamp examination and fundoscopy. In an exploratory tract anamnesis, the patient indicated that he suffered from loss of appetite and aversion to meat.

To determine the nature of the lesion, a biopsy of the tumor in the left rectus lateralis muscle was scheduled. The patient was also referred to the department of Internal Medicine because the case was suspected of a malignancy or metastasis of a yet unknown origin.

The physical and laboratory examination, including prostate-specific antigen, showed no abnormalities. Due to the high suspicion of malignancy, a CT of the neck, chest, and abdomen was performed which showed a space-consuming process at the level of the cardia with local lymphadenopathy. Additional endoscopy found a necrotizing swelling at the level of the cardia. Pathological examination of the gastric biopsy showed a moderately differentiated adenocarcinoma from the gastric epithelium (shown in Fig. 3). One week later, due to severe vertigo, the patient was admitted again, and a spinal tap and another MRI of the brain was performed. This showed malignant cells consistent with a metastasis of large cell carcinoma. The MRI also showed leptomeningeal metastases at the VII and VIII nerves on both sides.

In conclusion, the patient suffered from an adenocarcinoma of the stomach with lymphogenic and leptomeningeal metastasis and a swelling of the left rectus lateralis muscle which was highly suspected of metastasis. An orbita biopsy was omitted, as this would have no further clinical consequences. Partly at the request of the patient, it was decided to start best supportive care because of the poor prognosis. Unfortunately, the patient died 2 months after the first complaints started.

\section{Karger'}


Fig. 1. Transverse MRI section showing a solitary thickening of the left rectus lateralis muscle.
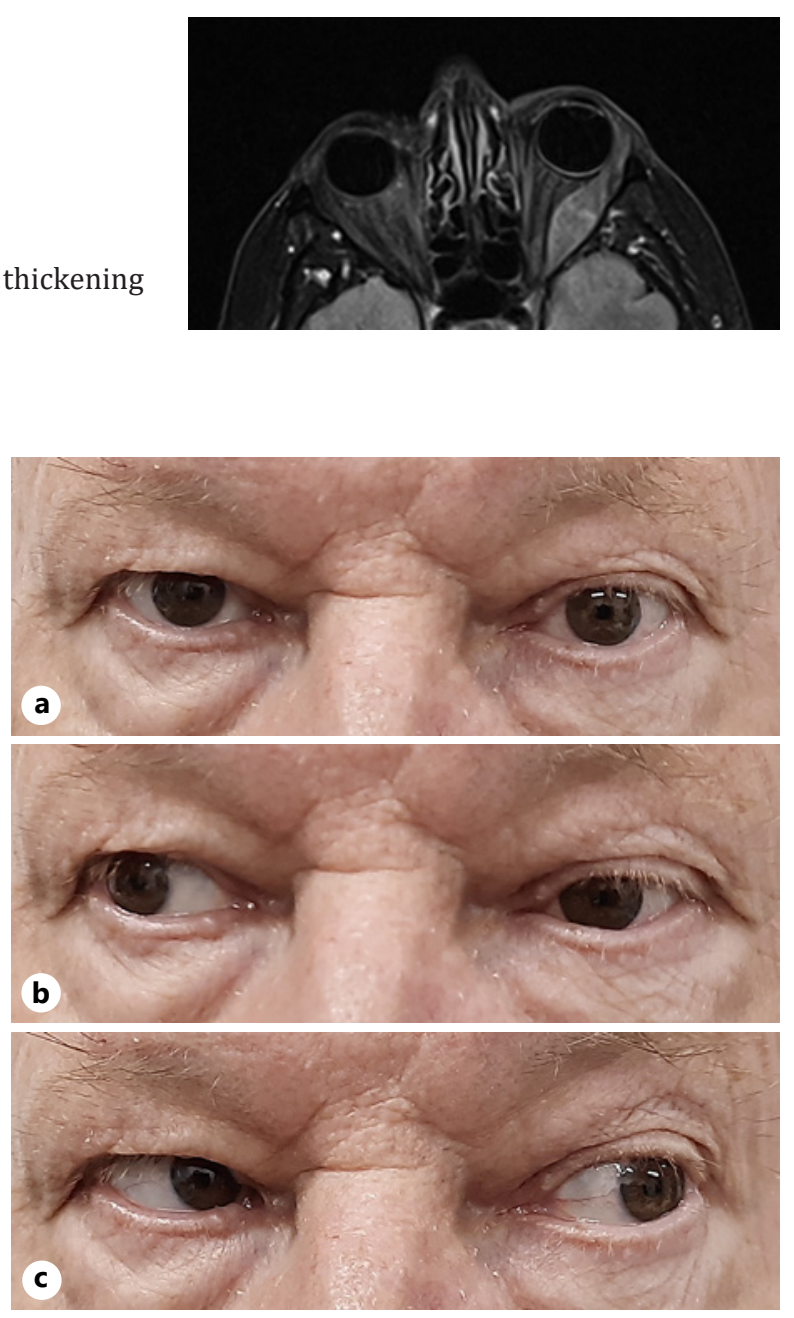

Fig. 2. Eye motility: primary position (a) and horizontal eye movements $(\mathbf{b}, \mathbf{c})$ are shown. The adduction of the left eye is abnormal (b).

Fig. 3. Pathology section showing tumor (upper part) and normal gastric tissue (bottom part). The normal tube structures are no longer recognizable in the tumor tissue.

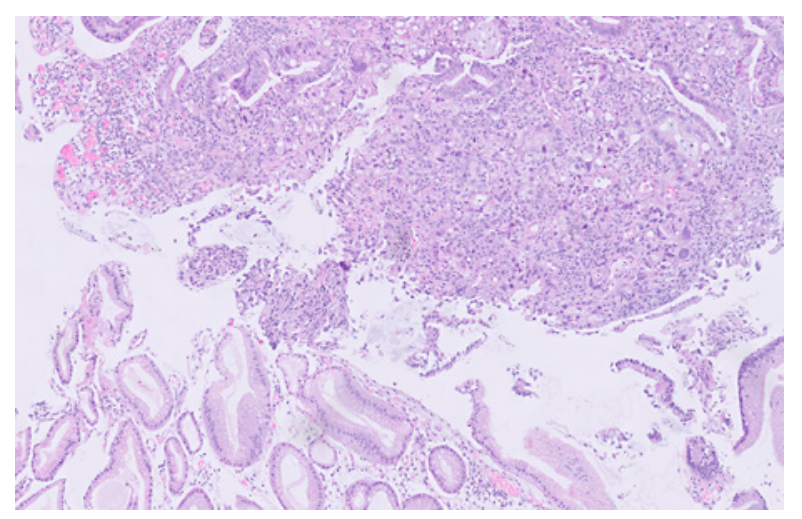

\section{Discussion}

Our case describes a rare metastatic pattern of gastric carcinoma involving intraorbital and leptomeningeal metastases. This patient illustrates that an orbital metastasis can be the initial manifestation of a yet undiagnosed malignancy of unknown origin. 
Nowadays, early recognition and improved treatment options of various malignancies increase life expectancy, increasing the incidence of metastases to unusual locations such as the orbit $[1,2]$. The estimated incidence of orbital metastases is $1-13 \%$ in (small) cohort studies $[3,4]$.

Of all intraorbital metastases, $25 \%$ concerns the initial manifestation of a yet undiagnosed primary malignancy [3]. Breast cancer is the most common cause of orbital metastasis $[1,4,5]$. Other described primary sites include the lung, prostate, gastrointestinal tract (including stomach), skin, adrenal gland, kidney, eye, and thyroid [1-7].

However, an orbital metastasis of a gastric carcinoma is extremely rare, and there is little literature about this so far $[8,9]$. The gastric carcinoma usually metastasizes to the local lymph nodes, peritoneum, liver, lung, bone, or adrenal gland. Metastasis to a skeletal muscle is even very rare [10].

Diplopia can be caused by an ophthalmic, neurological, or mechanical cause. In adults, the most common cause of diplopia is an optic nerve palsy due to ischemia. Other common causes include a cerebrovascular incident, intracerebral aneurysm, malignancy (intracerebral or intraorbital), and giant cell arteritis [11].

In addition to diplopia, proptosis, ptosis, vision loss, and pain may also be symptoms consistent with orbital metastasis [6]. The presentation is usually unilateral.

Initially, our patient was thought to have a primary neurological explanation for the complaints which was not illogical given the presentation. However, in retrospect, the aversion to meat turned out to be an important clue. Another essential clue was the fact that radiological imaging showed a solitary thickening in an extraocular muscle without any inflammatory features present. If this is the case, a primary malignancy or metastasis must always be ruled out. Furthermore, although it is logical to include a previously diagnosed malignancy (in this case the prostate carcinoma) in the differential diagnosis of a solitary ocular muscle swelling, this case shows that a metastasis from a second malignancy must also be taken into account. Based on a normal prostate-specific antigen level and pathological examination of the gastric tissue biopsy and spinal tap consistent with gastric carcinoma, a metastasis of prostate cancer in this case is highly unlikely, although we do not have a tissue biopsy of the orbital metastasis to prove this [12].

Treatment of orbital metastases is usually palliative and may include systemic therapy (chemotherapy, hormone therapy, or immunotherapy depending on the primary tumor), radiotherapy, and/or surgery [13]. The strategy should be determined by a multidisciplinary treatment team.

The prognosis for these patients is usually poor, as metastasis to the orbit and ocular adnexa usually indicates extensive hematogenous metastasis [1]. The innovation in therapy improves quality of life and preserves eye function more often $[1,14]$. The most optimal guidance and treatment for these patients can be realized through a multidisciplinary approach.

In conclusion, metastasis of a gastric carcinoma to the orbit is extremely rare. A solitary thickening in an extraocular muscle without inflammatory features is suspected of a tumor or metastasis. Always consider an orbital malignancy, with the extraocular muscles as the preferred location, in a patient with diplopia, proptosis, ptosis, vision loss, or pain.

\section{Statement of Ethics}

Written informed consent was obtained from the patient's family for publication of this case report and any accompanying images. This study protocol was reviewed and the need for approval was waived by the Research Committee of The Rotterdam Eye Hospital.

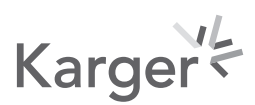


Roohé et al.: Diplopia as the First Sign of Gastric Carcinoma

\section{Conflict of Interest Statement}

The authors have no conflicts of interest to declare. All authors have confirmed this through the International Committee of Medical Journal Editors (ICMJE) Form for Disclosure of Potential Conflicts of Interest.

\section{Funding Sources}

The authors have no funding sources to declare. All authors have confirmed this through the ICMJE Form for Disclosure of Potential Conflicts of Interest.

\section{Author Contributions}

Authorship was based on the ICMJE criteria, including design and drafting of the manuscript, accountability for accuracy and integrity, and final approval. Suzanna L. Roohé and Dion Paridaens were responsible for the design, draft, and final version of the manuscript. Ivan M. Gan, Kim van der Weerd, Robert M. Verdijk, and Boaz Lopuhaä were responsible for the figures, revisions, and final version of the manuscript.

\section{Data Availability Statement}

All data generated or analyzed during this case report are included in this article and its figures. Further enquiries can be directed to the corresponding author.

\section{References}

1 Ahmad SM, Esmaeli B. Metastatic tumors of the orbit and ocular adnexa. Curr Opin Ophthalmol. 2007 Sep;18: 405-13.

2 Tan Z. Recent advances in the surgical treatment of advanced gastric cancer: a review. Med Sci Monit. 2019 May;25:3537-41.

3 Chen Y, Yang YC, Tang LY, Ge QM, Shi WQ, Su T, et al. Risk factors and their diagnostic values for ocular metastases in gastric adenocarcinoma. Cancer Manag Res. 2021 Mar;13:5835-43.

4 Vlachostergios PJ, Voutsadakis IA, Papandreou CN. Orbital metastasis of breast carcinoma. Breast Cancer. 2009 Dec;3:91-7.

5 Amemiya T, Hayashida H, Dake Y. Metastatic orbital tumors in Japan: a review of the literature. Ophthalmic Epidemiol. 2002 Feb; 9:35-47.

6 Karti O, Ozdemir 0, Top Karti D, Zengin MO, Tatli S, Kusbeci T, et al. Orbital metastasis secondary to breast cancer: a rare cause of unilateral external ophthalmoplegia. Neuroophthalmology. 2020 Jan;45:181-3.

7 Hanafi H, Verdijk RM, Paridaens D. Malignant pleural mesothelioma with lacrimal gland metastasis. Acta Ophthalmol. 2016 Dec;94:836-8.

8 Goto S, Takeda H, Sasahara Y, Takanashi I, Yamashita H. Metastasis of advanced gastric cancer to the extraocular muscle: a case report. J Med Case Rep. 2019 Apr;13:107-3.

9 Souayah N, Krivitskaya N, Lee HJ. Lateral rectus muscle metastasis as the initial manifestation of gastric cancer. J Neuroophthalmol. 2008 Sep;28(3):240-1.

10 Huang D-D, Lu J-X, Zhuang C-L, Yu Z, Zhang X-D. Metastasis to skeletal muscle from gastric adenocarcinoma: a case report and literature review. Int J Clin Exp Pathol. 2016;9(2):2414-8.

11 Peragallo JH, Newman NJ. Diplopia-an update. Semin Neurol. 2016 Aug;36(4):357-61.

12 Rima FA, Hussain M, Dewan RK, Haque MN, Sultana T, Chowdhury F, et al. Clinicopathologic features of gastric and gastrooesophageal junction adenocarcinoma. Mymensingh Med J. 2020 Jan;29(1):195-201.

13 Das M. Neoadjuvant chemotherapy: survival benefit in gastric cancer. Lancet Oncol. 2017 May;18(6):e307.

14 Shields JA, Shields CL, Brotman HK, Carvalho C, Perez N, Eagle RC. Cancer metastatic to the orbit: the 2000 Robert M. Curts lecture. Ophthalmic Plast Reconstr Surg. 2001 Sep;17:346-54. 